\title{
Análisis de la eficiencia técnica hospitalaria 2011
}

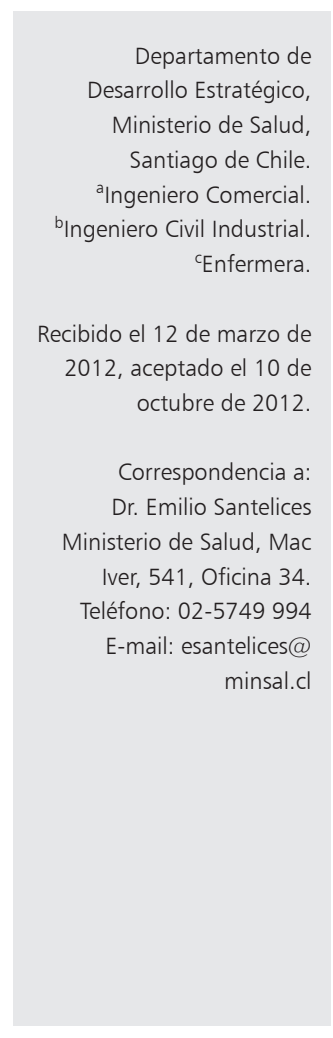

\author{
EMILIO SANTELICES C., HÉCTOR ORMEÑO C.a, \\ MAGDALENA DELGADO S. ${ }^{b}$, CHRISTOPHER LUI M. ${ }^{b}$, \\ RAÚL VALDÉS V. ${ }^{\mathrm{a},}$ LORENA DURÁN C. ${ }^{\mathrm{c}}$
}

\section{Analysis of hospital technical efficiency during 2011}

\begin{abstract}
Background: Efficiency in the use of resources in health systems and hospitals has been a matter of interest for administrators as well as for policy makers. The growing costs due to higher levels of demand from the population require a better use and allocation of such costs. Aim: To assess the technical efficiency in 28 hospitals in Chile, considering the period from May to October, 2011. Material and Methods: The average number of available beds, the number of staff and the expenses on consumer and service goods were used as production inputs and, on the other hand, the expenditures adjusted by the Diagnosis-Related Groups (IR-DRG) were used as products. To assess the technical efficiency, the Data Envelopment Analysis technique was used. Results: The levels of inefficiency fluctuate from 20 to $23.3 \%$. In other words, with a $20 \%$ increase in hospital discharges and maintaining fixed inputs, hospitals could become efficient. Conclusions: The incorporation of methodologies to determine efficiency allows gathering new knowledge for people who manage resources as well as for policy makers by optimizing practices and having better allocation criteria.
\end{abstract}

(Rev Med Chile 2013; 141: 332-337).

Key words: Data collection; Data interpretation, statistical; Diagnosis-related groups; Hospitals.

E 1 análisis de la eficiencia constituye un objetivo central de los hacedores de política en los sistemas de salud. El gasto en salud como porcentaje de la producción se ha ido incrementando y han aparecido nuevos desafíos, propios de los cambios epidemiológicos y el envejecimiento que enfrentan los países en desarrollo. Parte importante de los recursos en salud se consumen en los hospitales, por lo que la mayoría de los investigadores ha concentrado sus esfuerzos de análisis a ese nivel.

El análisis de la eficiencia hospitalaria se ha venido desarrollando desde la década 1980-89 a través de una herramienta denominada Data Envelopment Analysis ${ }^{1}$. El enfoque de este trabajo dice relación con la "eficiencia técnica", la que corresponde a la capacidad que tiene una unidad de producción, de ocupar el mínimo de insumos para generar una cantidad dada de producto (orientación input), o de la misma forma, generar el máximo de unidades de producto a partir de una determinada cantidad de insumos (orientación output $)^{2}$.

Dentro de los resultados de la literatura internacional se observa que en la mayoría de los casos existen porcentajes de ineficiencia ${ }^{39}$. La mayoría de estos espacios de ineficiencia se deben a que se realiza un análisis comparativo entre las diferentes unidades productivas, y es altamente probable que cada unidad tenga sus propias prácticas de gestión y diferente tecnología. De ahí la importancia de estimar la eficiencia, pues implica conocer aquellos establecimientos que se encuentran en la frontera de eficiencia, y permitir replicar las buenas prácticas.

En Chile, destacan los estudios de Castro 
$(2004)^{10}$ y Barahona-Urbina $(2011)^{11}$. El primero estima la producción considerando para ello como insumos el gasto total del hospital, el número de camas por hospital, y como productos, el promedio de días de estada, y el número de consultas de especialidad y de urgencia. Los resultados indican que sólo $16 \%$ de los hospitales se encuentra operando en niveles eficientes de producción, y se utilizan entre $30,3 \%$ y $94,3 \%$ más de recursos. El segundo estudio usa como variables de insumo el personal médico, enfermeras y matronas, y como producto el egreso hospitalario. Dentro de los resultados se tiene que los hospitales deberían reducir sus insumos de producción en 10\% para situarse en la frontera eficiente, y señala que esta ineficiencia puede estar explicada por el sobredimensionamiento y el costo del recurso humano.

El objetivo de este trabajo es evaluar la eficiencia técnica de 28 hospitales para el período mayo a octubre de 2011 usando la técnica Data Envelopment Analysis (DEA), usando para ello variables de insumo y producto hospitalario. Este trabajo se circunscribe a la actividad hospitalaria por lo que los aspectos relacionados con la actividad ambulatoria, tanto en resultados como en insumos utilizados han sido excluidos. En las siguientes secciones se especifica la metodología del estudio, los resultados y discusión.

\section{Material y Método}

La muestra corresponde a 28 hospitales, los cuales concentran la cantidad de 255.439 egresos para el período mayo a octubre de 2011, y que representan $45 \%$ de los egresos totales a nivel nacional para el mismo período.

Para aplicar la técnica DEA es necesario definir un set de insumos y productos. La mayoría de los estudios usa como aproximaciones del insumo medidas de capital y trabajo, y como productos, los días de hospitalización y los egresos, existiendo una tendencia generalizada a la corrección por el case-mix ${ }^{1}$. El case-mix corresponde a una medida de la complejidad de la enfermedad del paciente, así como al tratamiento asociado.

\section{Variables de insumo}

Respecto a los insumos se usarán variables relacionadas al trabajo, al capital, y también al consumo de recursos. Respecto a las primeras, se tiene la dotación de "personal de leyes médicas" y la dotación de "personal del estatuto administrativo" a junio de 2011. Dicha información es suministrada por la División de Gestión y Desarrollo de las Personas, Ministerio de Salud.

El "personal de leyes médicas" incluye al número de médicos, odontólogos, químicos farmacéuticos, bioquímicos, y también el personal que se encuentra haciendo becas o devolviendo su período asistencial, siendo los médicos $83 \%$ de este personal. Respecto al personal de estatuto, se tienen los directivos, asistentes sociales, enfermeras, matronas, fonoaudiólogos, kinesiólogos, nutricionistas, psicólogos, otros profesionales, tecnólogos médicos, terapeutas, otros técnicos, administrativos y auxiliares, siendo los mayores porcentajes para el caso de los técnicos paramédicos $(28 \%)$, los administrativos y auxiliares $(30 \%)$, las enfermeras (11\%), técnicos de nivel superior (9\%), y matronas $(4 \%)$.

Respecto al capital, o aquella variable que determina la producción en el mediano-largo plazo, la mayoría de los estudios usa las camas hospitalarias $^{3,5,6,8,10,11}$. En este caso, se tiene el promedio de camas disponibles para el período mayo-octubre de 2011, disponible en la base de datos del Departamento de Estadísticas e Información de Salud, del Ministerio de Salud. Se considera el promedio pues las camas disponibles no varían significativamente de mes a mes.

Diversos estudios usan los gastos para aproximar el consumo de recursos ${ }^{8,10}$. En este estudio usamos los bienes y servicios de consumo (Subtítulo 22 del Clasificador Presupuestario), los que corresponden al gasto por concepto de productos farmacéuticos, materiales y útiles quirúrgicos, alimentos y bebidas, combustibles y lubricantes, y otros insumos necesarios para el normal funcionamiento del hospital. Dichos gastos se tienen para el período mayo-octubre de 2011 y se obtienen de los costos que los hospitales informan mes a mes al Departamento de Desarrollo Estratégico del Ministerio de Salud.

\section{Variables de producto}

El proceso hospitalario consiste en una agregación de actividades hospitalarias en un paciente, y culmina con el alta hospitalaria ${ }^{12}$. La necesidad de una medición específica y sintética nos lleva a elegir al egreso como el producto hospitalario, el cual se obtiene para el período mayo a octubre de 
2011, del Departamento de Estadísticas de Información de Salud, del Ministerio de Salud.

Sin embargo, es necesario corregir por complejidad, pues el egreso de un paciente cuyo tratamiento es simple y que no tiene complicaciones asociadas, no tiene el mismo impacto que un paciente de alta complejidad. Para ponderar los egresos se usa el peso relativo de los Grupos Relacionados por el Diagnóstico (IR-GRD) tal como se describe a continuación. Si se tienen 4 casos de resección colorrectal y 2 casos de histerectomía, y el peso relativo del GRD para la resección colorrectal es 3,4 y para la histerectomía es 0,8 , entonces los egresos ponderados serian $4 \times 3,4+2 \times 0,8=15,2^{13}$.

Existen autores que señalan que el omitir las consultas puede llevar a sesgar los costos de los hospitales ${ }^{14}$, sin embargo, en nuestro caso, se tiene que las consultas tienen un elevado grado de correlación con los egresos hospitalarios. De acuerdo a estimaciones del Departamento de Desarrollo Estratégico, el coeficiente de correlación de Pearson entre los egresos y consultas de cada establecimiento para el período mayo-octubre de 2011 alcanza la cifra de $90,7 \%(\mathrm{p}<0,01)$. Por otro lado, el egreso representa $60 \%$ de los costos totales de los hospitales, y una simple estimación de costos medios de egresos y costos medios de consultas a partir de nuestros datos señala que el primero es 5,84 veces el segundo.

Esta elección de insumos y productos no se encuentra alejada de la definición que han hecho la mayoría de los estudios relacionados a la eficiencia hospitalaria ${ }^{1,5,8,15}$, los cuales optan por considerar a veces como insumos, el salario, el gasto total, o como producto, las consultas o los días de hospitalización entre otros indicadores.

\section{Data Envelopment Analysis}

La técnica DEA corresponde a una herramienta no paramétrica y determinista, que a través de programación matemática estima una frontera de eficiencia, para posteriormente obtener índices de eficiencia de cada unidad productiva. El que sea no paramétrica implica que no es necesario especificar una forma funcional de la función frontera, mientras que el carácter determinista implica la no presencia de un componente aleatorio ${ }^{1,2}$.

Una vez estimada la frontera de eficiencia, se obtienen índices de cada unidad productiva respecto de dicha frontera. En términos de la Figura 1 , se tienen dos insumos, $Y_{1}$ y $Y_{2}$, para producir
$X$. A partir de las unidades que tienen una mayor productividad se construye la frontera de eficiencia, $Z Z$ '. Entonces, el índice de eficiencia técnica de $\mathrm{A}\left(E T_{A}=O A / O B<1\right)$ es inferior a 1 , mientras que el índice de eficiencia técnica de $\mathrm{B}\left(E T_{B}=O B /\right.$ $O B=1$ ) es igual a 1 , lo que implica que la unidad $B$ es técnicamente eficiente.

De acuerdo a lo anterior, el resultado de la aplicación del modelo es un índice de eficiencia que se encuentra entre "0" 0 " 1 ", donde 1 corresponde a la cifra máxima de eficiencia y representa a aquellos hospitales que dado un nivel de insumos producen lo máximo posible, o a aquellos que dado un nivel de producción están usando la menor cantidad de recursos.

El modelo a aplicar será el DEA output orientado, el que implica que las unidades productivas maximizarán la producción por cada unidad de insumo. Una gran parte de los estudios utiliza la modalidad input orientado, que presupone que las unidades productivas minimizan sus costos por unidad de producto ${ }^{8,10,11,15}$ asumiendo que los egresos son en gran parte exógenos al hospital, pues dependen de la demanda por prestaciones de salud.

Sin embargo, si bien es cierto que la demanda por prestaciones es de carácter exógena al hospital, pues el hospital no puede influir en su origen, se asume que la eficiencia en la gestión de los egresos es de carácter endógena al hospital, por lo que la mayor eficiencia en cada uno de los procesos que implican un egreso hospitalario se debería reflejar en una mayor productividad. Asimismo, dado que las camas disponibles y el personal se mantienen

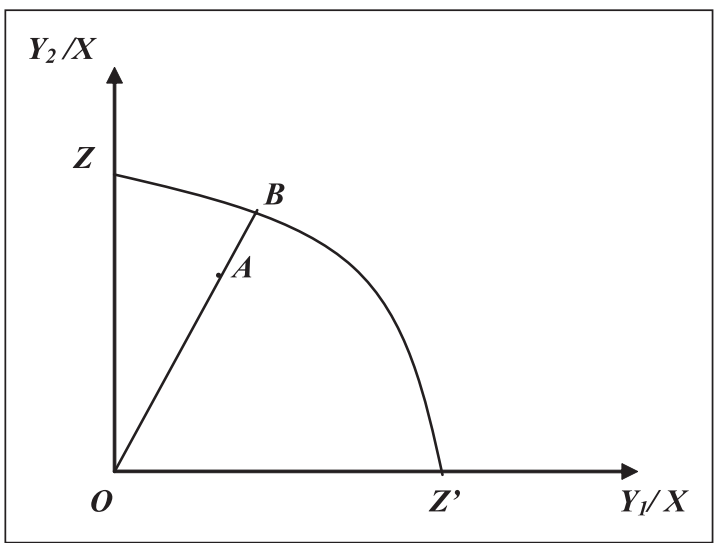

Figura 1. Frontera de Eficiencia: Dos productos, $Y_{1}$ y $Y_{2}$, y un insumo, $X$ (En imagen). Fuente: elaboración propia. 
fijos por un período de tiempo considerable, resulta adecuado ocupar este supuesto.

A su vez es necesario destacar que la estimación del $D E A$ se puede llevar a cabo a través del supuesto de retornos constantes $\left(D E A_{\text {const }}\right)$ o variables a la escala $\left(D E A_{v a r}\right)$. El primer supuesto implica que el aumento de $\mathrm{x} \%$ en los insumos implica un aumento de $\mathrm{x} \%$ en el producto final, mientras que el segundo supuesto implica que el mismo aumento en los insumos produce un aumento menor a $\mathrm{x} \%$ en la producción final. La obtención de dichos índices permite obtener la eficiencia a la escala $\left(D E A_{\text {scale }}\right)$, o la eficiencia imputable al tamaño de producción de los hospitales ( $D E A_{\text {const }}$ $\left./ D E A_{\text {var }}=D E A_{\text {scale }}\right)$.

La estimación de los modelos DEA se realiza a través del software DEAP versión 2.1.

\section{Resultados}

Los resultados de la aplicación del DEA se observan en la Tabla 1, donde se encuentra la eficiencia bajo retornos constantes a la escala, a retornos variables, y la eficiencia técnica a escala (detalles en Anexo, Tabla 3).

De acuerdo a la Tabla 1, el promedio de eficiencia asumiendo retornos constantes fue de $76,7 \%$, lo que implica que los hospitales produciendo 23,3\% más de egresos dados los insumos constantes, podrían aumentar sus niveles de eficiencia. El hospital más ineficiente en este modelo podría producir $53,7 \%$ más egresos sin modificar los insumos de producción. Los hospitales que se encuentran produciendo en la frontera de eficiencia corresponden al 21\% de la muestra.

Sin embargo, el supuesto de rendimientos constantes puede ser demasiado fuerte en la realidad, pues el tamaño y el incremento en la complejidad del hospital, podría incidir en que el aumento de una unidad de insumo no tenga como efecto un aumento en la misma cuantía en la producción.

De acuerdo a lo anterior, es necesario aplicar el modelo DEA bajo el supuesto de retornos variables. De acuerdo a este modelo, los hospitales tienen capacidad ociosa en torno al 20,2\%, mientras que el peor evaluado podría aumentar la producción en $52,2 \%$ sin modificar sus insumos para situarse en la frontera de eficiencia. A su vez, $32 \%$ de los hospitales se encontraba operando en la frontera de eficiencia.
Tabla 1. Resultados Eficiencia Técnica, rendimientos constantes y variables

\begin{tabular}{|lccc|}
\hline & $\begin{array}{c}\text { Eficiencia } \\
\text { R. } \\
\text { Constantes }\end{array}$ & $\begin{array}{c}\text { Eficiencia } \\
\text { R. } \\
\text { Variables }\end{array}$ & $\begin{array}{c}\text { Eficiencia } \\
\mathbf{R .} \\
\text { Escala }\end{array}$ \\
Media & 0,767 & 0,798 & 0,963 \\
Mediana & 0,728 & 0,762 & 0,988 \\
\hline Mínimo & 0,463 & 0,478 & 0,763 \\
Máximo & 1,000 & 1,000 & 1,000 \\
\hline Desviación estándar & 0,169 & 0,175 & 0,060 \\
Coef. de variación & $22 \%$ & $22 \%$ & $6 \%$ \\
\hline Hosp. en la Frontera & $21 \%$ & $32 \%$ & $21 \%$ \\
\hline
\end{tabular}

Fuente: elaboración propia en base a estimaciones DEA.

Tabla 2. Indicadores de insumo productividad y estado de eficiencia de hospitales

\begin{tabular}{|c|c|c|}
\hline Indicador & $\mathrm{DE}_{\text {Avar }}=1$ & $\mathrm{DE}_{\text {Avar }}<1$ \\
\hline Costo por egreso & $931,939.2$ & $1,070,203$ \\
\hline Bienes y Scios. por egreso & $361,143.9$ & $423,462.3$ \\
\hline $\begin{array}{l}\text { Egreso por enfermera o } \\
\text { matrona }\end{array}$ & 58,32 & 47,62 \\
\hline Egreso por cama disponible & 27,71 & 24,25 \\
\hline
\end{tabular}

Fuente: elaboración propia en base a estimaciones DEA. Costos, Bienes y servicios, y Egresos, período mayo a octubre de 2011. Enfermeras a junio de 2011. Camas corresponden a promedio período mayo a octubre de 2011 .

Por otro lado, se tiene la eficiencia imputable al tamaño de los hospitales, o la ineficiencia a escala. Como se observa, los hospitales producían $3,7 \%$ menos debido a que son muy grandes o muy pequeños en relación al tamaño óptimo de producción.

Luces respecto a las causas de la ineficiencia pueden deducirse del análisis de la Tabla 2, en la cual se han escogido indicadores de la productividad por insumo, respecto de si el establecimiento se encuentra en la frontera de eficiencia o fuera de ella $(D E A)$

De la Tabla 2 se infiere que aquellos hospitales que se encuentran en la frontera de eficiencia tienen un costo de egreso $12,92 \%$ menor en relación a aquellos que no están en la frontera, al igual que el caso de los bienes y servicios, que están por debajo en $14,72 \%$.

Respecto de las enfermeras, que se eligen por 
Tabla 3. Resultados Eficiencia Técnica, rendimientos constantes y variables

\begin{tabular}{|c|c|c|c|}
\hline Hospital & $\begin{array}{c}\text { Eficiencia } \\
\text { R. Constantes }\end{array}$ & $\begin{array}{l}\text { Eficiencia } \\
\text { R. Variables }\end{array}$ & $\begin{array}{l}\text { Eficiencia } \\
\text { R. Escala }\end{array}$ \\
\hline Complejo Asistencial Dr. Víctor Ríos Ruiz (Los Angeles) & 0,734 & 0,736 & 0,997 \\
\hline Complejo Hospitalario Dr. Sótero del Río (Santiago, Puente Alto) & 1,000 & 1,000 & 1,000 \\
\hline Complejo Hospitalario San José (Santiago, Independencia) & 0,897 & 0,913 & 0,982 \\
\hline Hospital Barros Luco Trudeau (Santiago, San Miguel) & 0,528 & 0,528 & 0,999 \\
\hline Hospital Carlos Van Buren (Valparaíso) & 0,974 & 1,000 & 0,974 \\
\hline Hospital Clínico de Niños Dr. Roberto del Río (Santiago, Independencia) & 0,586 & 0,630 & 0,931 \\
\hline Hospital Clínico Herminda Martín (Chillán) & 0,658 & 0,667 & 0,987 \\
\hline Hospital Clínico Regional (Valdivia) & 0,634 & 0,636 & 0,996 \\
\hline Hospital de Castro & 0,901 & 1,000 & 0,901 \\
\hline Hospital de Niños Dr. Luis Calvo Mackenna (Santiago, Providencia) & 1,000 & 1,000 & 1,000 \\
\hline Hospital de Urgencia Asistencia Pública Dr. Alejandro del Río (Santiago) & 0,600 & 0,607 & 0,989 \\
\hline Hospital del Salvador (Santiago, Providencia) & 0,688 & 0,689 & 0,998 \\
\hline Hospital Dr. Antonio Tirado Lanas (Ovalle) & 1,000 & 1,000 & 1,000 \\
\hline Hospital Dr. Ernesto Torres Galdames (Iquique) & 0,687 & 0,694 & 0,990 \\
\hline Hospital Dr. Exequiel González Cortés (Santiago, San Miguel) & 0,763 & 1,000 & 0,763 \\
\hline Hospital Dr. Félix Bulnes Cerda (Santiago, Quinta Normal) & 0,463 & 0,478 & 0,970 \\
\hline Hospital Dr. Gustavo Fricke (Viña del Mar) & 0,686 & 0,708 & 0,968 \\
\hline Hospital Dr. Hernán Henríquez Aravena (Temuco) & 0,621 & 0,648 & 0,958 \\
\hline Hospital Dr. Juan Noé Crevanni (Arica) & 0,805 & 0,837 & 0,962 \\
\hline Hospital Dr. Luis Tisné B (Santiago, Peñalolén) & 0,875 & 0,998 & 0,877 \\
\hline Hospital Dr. Mauricio Heyermann (Angol) & 1,000 & 1,000 & 1,000 \\
\hline Hospital El Pino (Santiago, San Bernardo) & 1,000 & 1,000 & 1,000 \\
\hline Hospital Regional (Coihaique) & 0,632 & 0,792 & 0,797 \\
\hline Hospital Regional de Rancagua & 0,721 & 0,730 & 0,988 \\
\hline Hospital San Juan de Dios (Curicó) & 0,752 & 0,787 & 0,956 \\
\hline Hospital San Juan de Dios (Santiago, Santiago) & 0,551 & 0,557 & 0,991 \\
\hline Hospital San Pablo (Coquimbo) & 0,710 & 0,720 & 0,986 \\
\hline Instituto Nacional de Enfermedades Respiratorias y Cirugía Torácica & 1,000 & 1,000 & 1,000 \\
\hline Promedio & 0,767 & 0,798 & 0,963 \\
\hline
\end{tabular}

Fuente: elaboración propia en base a estimaciones DEA.

su gran responsabilidad en la gestión hospitalaria, aquellos establecimientos en la frontera tienen un mayor valor de egresos por enfermera, al igual que el caso de los egresos por cama disponible.

\section{Discusión}

Los resultados de este estudio muestran cifras de ineficiencia relativa cercanos al 23,3-20,2\%, donde los hospitales podrían aumentar la producción en estas magnitudes manteniendo los insumos constantes. Cabe señalar que este rango se da pues se estimaron modelos DEA suponiendo retornos constantes y variables a la escala. La eficiencia por tamaño de escala no tiene un valor significativamente importante.

La elección de los egresos ponderados como indicador sintético de producto, implica que existen 
establecimientos que tienen una mayor productividad en términos de todos los procedimientos y servicios que implica ese egreso, y la eficiencia a su vez es relativa, pues depende de las diferentes prácticas existentes en el sector hospitalario. Además, esta mayor eficiencia en los egresos implica un mejor uso de los recursos y un mayor acceso para toda la población.

Adicionalmente, no se ha hecho una separación de tipologías entre hospitales, pues la cantidad de pacientes ingresados a cada uno depende de características exógenas al hospital, por el contrario, la cantidad de recursos gastados en cada egreso es una variable endógena al hospital, sobre la cual recae el foco de este trabajo.

La información que aporta esta evidencia a los tomadores de decisiones constituye una variable adicional, que permite mediante la comparación de diferentes hospitales, conocer cuáles son los que se ubican en la frontera de eficiencia, y asumir por parte de los otros las mejores prácticas que estos representan con el propósito de optimizar el uso de sus recursos. No debe utilizarse como una aproximación única para calificar o asumir determinaciones de política, sino como una forma de incorporar nuevas metodologías que contribuirán a mejorar el quehacer hospitalario y la toma de decisiones en torno al uso de sus recursos.

\section{Referencias}

1. Hollingsworth B. The measurement of efficiency and productivity of health care delivery. Health Economics 2008; 17 (10): 1107-1128.

2. Farrel MJ. The Measurement of Productive Efficiency. Journal of the Royal Statistical Society Series 1957; 120 (3): 253-278.

3. Ozcan YA, Luke RD, Haksever C. Ownership and Organizational Performance: A Comparison of Technical Efficiency across Hospital Types. Medical Care 1992; 30 (9): 781-94.

4. Ferrier G, Valdmanis V. Rural hospital performance and its correlates. The Journal of Productivity Analysis 1996; 7 (1): 63-80.

5. Hofmarcher M, Paterson I, Riedel M. Measuring Hospital Efficiency in Austria-A DEA Approach. Health Care Management Science 2002; 5 (1): 7-14.

6. Gannon B. Technical Efficiency of Hospitals in Ireland. Working paper, Economic and Social Research Institute
(ESRI) 2004; 18: 1-31.

7. Laine J, Finne-Soveri H, Bjorkgren M, Linna M, Noro A, Hakkinen $\mathrm{U}$. The association between quality of care and technical efficiency in long-term care. International Journal for Quality in Health Care 2005; 17 (3): 259267.

8. Nayar P, Ozcan YA. Data envelopment analysis comparison of hospital efficiency and quality. Journal of Medical Systems 2008; 32 (3): 193-9.

9. Seijas A, Iglesias G. (2009). Medida de la Eficiencia Técnica en los Hospitales Públicos Gallegos. Revista Galega de Economía 2009; 18 (1): 1-22.

10. Castro R. Midiendo la (in)eficiencia de los hospitales públicos en Chile. Libertad y Desarrollo, Informe Social 2004; 83: 1-52.

11. Barahona-Urbina P. Análisis de eficiencia hospitalaria en Chile. An Fac Med 2011; 72 (1): 33-38.

12. Temes, JL. Gestión hospitalaria. Madrid, España: Editorial MacGraw-Hill-Interamericana; 2002. Tercera edición. p. 25.

13. Dexter F, O'Neill L. Data envelopment analysis to determine by how much hospitals can increase elective inpatient surgical workload for each specialty. Anesth Analg. 200499 (5): 1492-500.

14. MacLean MB, Mix P. Measuring hospital productivity and output: the omission of outpatient services. Health Rep. 1991; 3 (3): 229-44.

15. Ligarda J, Naccha M. La eficiencia de las organizaciones de salud a través del análisis envolvente de datos. Microrredes de la dirección de salud IV Lima Este 2003. Anales de la Facultad de Medicina, Universidad Nacional Mayor de San Marcos 2006; 67 (2): 142-51.

16. Cid C, Ibern P. Regulación del financiamiento a Hospitales: "yardstick competition" aplicada a los hospitales públicos en Chile. Cuadernos Médico Sociales 2008; 48 (3): 155-64.

\section{Bases de datos}

Bases de Datos WinSIG, Costos hospitalarios, 2011. Departamento de Desarrollo Estratégico, Ministerio de Salud, Chile.

Dotación de Personal de establecimientos de Salud (2011), División de Gestión y Desarrollo de las Personas. Ministerio de Salud.

Ministerio de Salud, Departamento de Estadísticas de Información de Salud (2011).

Sistema ALCOR, IR-GRD (2011). Departamento de Desarrollo Estratégico, Ministerio de Salud, Chile. 